\title{
Prueba comparativa de uniformidad de contenido en tabletas de teofilina (150 mg/tab) de dos casas farmacéuticas en Costa Rica
}

\author{
Comparative test of content uniformity of \\ theophylline ( $50 \mathrm{mg} / \mathrm{tab}$ ) tablets from two \\ pharmaceutical houses in Costa Rica
}

\author{
Esteban Pérez-López' \\ Kevin Morales-Alfaro ${ }^{2}$ \\ Alfonso Rojas-Hernández ${ }^{3}$ \\ Anderson Vargas-Vargas ${ }^{4}$
}

Fecha de recepción: 22 de julio del 2013

Fecha de aprobación: II de octubre del 2013

Pérez-López, E; Morales-Alfaro, K; Rojas-

Hernández, A; Vargas-Vargas, A. Prueba comparativa de uniformidad de contenido en tabletas de teofilina ( $150 \mathrm{mg} / \mathrm{tab}$ ) de dos casas

farmacéuticas en Costa Rica. Tecnología en Marcha. Vol. 27, № 2. Pág 5I-57

I. Máster en Sistemas Modernos de Manufactura y bachiller en Laboratorista Químico. Profesor Universidad de Costa Rica. Costa Rica. Correo electrónico: esteban.perezlopez@ucr.ac.cr.

2. Estudiante de bachillerato en Laboratorista Químico. Universidad de Costa Rica. Costa Rica. Correo electrónico: kevin.morales@ucr.ac.cr.

3. Estudiante de bachillerato en Laboratorista Químico. Universidad de Costa Rica. Costa Rica. Correo electrónico: alfonso.rojas@ucrac.cr.

4. Estudiante de bachillerato en Laboratorista Químico. Universidad de Costa Rica. Costa Rica. Correo electrónico: anderson.vargas@ucr.ac.cr. 


\section{Palabras clave}

Teofilina; uniformidad de contenido; principio activo; tabletas; absorbancia; espectroscopía.

\section{Resumen}

Dada la importancia del medicamento denominado teofilina para su uso en pacientes con problemas de asma y su alto consumo en Costa Rica, se eligió el producto en tabletas de $150 \mathrm{mg}$ de teofilina de una casa farmacéutica que lo produce y distribuye en forma genérica, junto con el que fabrica la Caja Costarricense de Seguro Social (CCSS) en la misma dosis. Se realizó el estudio comparativo de la prueba de uniformidad de contenido para las tabletas de teofilina de $150 \mathrm{mg}$ de la casa farmacéutica LISAN y las fabricadas por la CCSS. La prueba de uniformidad de contenido analíticamente se fundamentó en la absorción y cuantificación del ingrediente activo teofilina a $272 \mathrm{~nm}$ para 10 muestras de cada casa farmacéutica, mediante el uso de un espectrofotómetro UVNis, empleando como disolvente agua destilada. Se probó un método selectivo, exacto y preciso y se obtuvo como resultado que todas las dosis ensayadas para cada fabricante se encuentran en el rango de $100 \%$ a $110 \%$, con respecto a lo etiquetado sobre el principio activo en las tabletas y con un desvío relativo no mayor al 2,5\%, cumpliendo holgadamente con lo establecido por la Farmacopea de Estados Unidos para la prueba de uniformidad de contenido.

\section{Key words}

Theophylline; content uniformity; active ingredient; tablets; absorbance; spectroscopy.

\begin{abstract}
Given the importance of the drug called theophylline, for use in patients with asthma, and given the high consumption of this drug in our country, we chose the product in tablets of $150 \mathrm{mg}$ of theophylline from a pharmaceutical company that produces and distributes in the form generic that drug, along with manufactured by the Social Security Fund (CCSS) at the same dose of the same drug. We performed a comparative study of content uniformity test for theophylline tablets $150 \mathrm{mg}$, pharmaceutical house LISAN and the Social Security Fund. The content uniformity test was based on the absorption of the active ingredient and theophylline quantification at $272 \mathrm{~nm}$ for 10 samples of each house pharmaceutical, by using a UVNVis, using distilled water as solvent. Proved that the method is selective, accurate and precise, and as a result was obtained that at all doses tested for each manufacturer, are in the range of $100 \%$ to $110 \%$, with respect to labeling of the active ingredient in the tablets and with a relative deviation of no more than $2,5 \%$, complying with the provisions of the Pharmacopoeia of the United States, to test for uniformity of content.
\end{abstract}

\section{Introducción}

La prueba de uniformidad de contenido es "el examen para determinar la variabilidad de cantidad del principio activo presente en unidades que sirven de muestra de un determinado lote" (Durán 201 I, p. 33)

La uniformidad de contenido se aplica principalmente a las formas farmacéuticas sólidas orales (tabletas y cápsulas), con el fin de medir el grado de variabilidad en el proceso de manufactura del medicamento $y$, aunque se puede aplicar en todos los casos, en términos generales se aplica a medicamentos en los que el contenido de principio activo es menor a $25 \mathrm{mg}$, o en aquellos casos en los que la cantidad de principio activo representa menos del $25 \%$ del tamaño total de la dosis en cuanto a su peso.

La prueba consiste en analizar diez dosis individuales del fármaco y determinar el contenido de principio activo presente en cada una de ellas. Cada dosis debe estar comprendida en el rango de 85-1।5\% de lo etiquetado en el medicamento y con una desviación no mayor al 6\%.

Según la Farmacopea de Estados Unidos, si I unidad está fuera del intervalo de 85,0\% a |15,0\% de la cantidad declarada en la etiqueta y ninguna unidad está fuera del intervalo de 75,0\% a 125,0\% de la 
cantidad declarada, o si el RSD es más de 6,0\%, o si ambas condiciones prevalecen, se analizan 20 unidades adicionales. Los requisitos se cumplen si no más de I unidad de las 30 está fuera del intervalo de $85,0 \%$ a $\mid 15,0 \%$ de la cantidad declarada y ninguna unidad está fuera del intervalo de $75,0 \%$ a $125,0 \%$ de la cantidad declarada y el RSD de 30 unidades de dosificación no es más de 7,8\%.

Las buenas prácticas de laboratorio han logrado fomentar y corroborar que un medicamento cumpla con las especificaciones del fabricante. Esto se hace por medio de métodos válidos de análisis para garantizar la eficacia, seguridad y homogeneidad de un medicamento, obteniéndose resultados fiables y documentados desde la práctica analítica (Lavaut, 2009).

La investigación se basó en el estudio de la teofilina, que se define como un fármaco muy empleado en la medicina humana en diversidad de formas farmacéuticas: tabletas, cápsulas, solución líquida y jarabe para tomar por vía oral. Se administra por vía oral y sufren procesos de liberación 3 a 4 veces al día.

La teofilina se presenta en variados medicamentos sola 0 asociada para el tratamiento de diferentes afecciones pulmonares (Ponce de León et al., 2005). La teofilina se usa para prevenir y tratar resfríos, falta de aire y dificultad para respirar causada por asma, bronquitis crónica, enfisema y otras enfermedades pulmonares (Kadam et al., 2009). La estructura de la teofilina se ilustra en la figura I.

El método analítico empleado para la determinación de uniformidad fue la espectroscopía ultravioleta. Refiriéndose a la espectroscopía para el análisis del principio activo, es de gran ayuda utilizar el método de espectrofotometría ultravioleta, ya que en las últimas décadas las técnicas de derivación por espectrofotometría han ganado rápidamente aplicaciones en el campo del análisis farmacéutico, superando problemas de interferencia, debido a la sustancia y el analito, comúnmente presenta formulaciones analíticas o la combinación de dos o más drogas (Bosch, 20I2).

Se prefiere la espectrofotometría ultravioleta, ya que se busca la cuantificación de un principio activo, buscando un análisis más sencillo eliminando el traslape de picos que presenta la espectroscopía infrarroja (Bosch, 20I2).
La investigación se basó en la cuantificación individual del ingrediente activo de un número de tabletas de teofilina mediante la técnica analítica de espectroscopía ultravioleta, analizando el producto de dos casas farmacéuticas (LISAN y la Caja Costarricense del Seguro Social, CCSS). Además se realizaron las pruebas estadísticas pertinentes para determinar si el ingrediente activo de las muestras se encuentra dentro de los límites establecidos por la Farmacopea de Estados Unidos (USP), que son 85-1 15\%. Además se determinó si hay una diferencia significativa en el contenido de teofilina en el producto entre los fabricantes.

La razón esencial de la escogencia de este medicamento es el efecto negativo que tiene sobre el cuerpo, ya que presenta efectos secundarios. En el caso de la teofilina, tiene una alta posibilidad de enlazarse con las moléculas de ADN (Johnson, Malathi, Prakash, Prathiba y Raghunathan 20 2, p. 4), lo cual conlleva una serie de problemas.

"Interesantemente, esos derivados de xantinas tienen interacciones con complejos esteroide-receptor, ADN, ARN, receptores de adenosina, quinasas de proteínas, y comportamiento neurológico, los cuales son contados para ser esenciales por su habilidad de modular las reacciones bioquímicas mediante la interacción con ácidos nucleicos o a través de moléculas de señalización celular" (Johnson et al. 2012, p. I).

Debido a ello, se han realizado determinaciones de concentración de teofilina dentro del cuerpo, ya que es un factor importante para establecer el

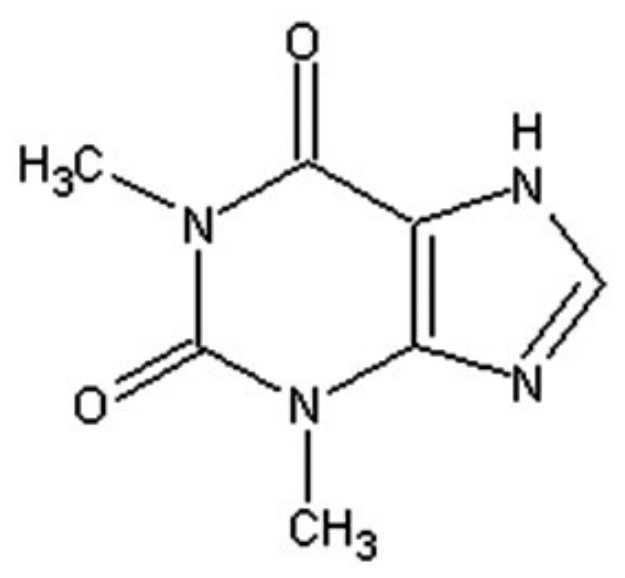

Figura I. Estructura de la teofilina. 
efecto que tendría en el organismo. Así lo indica Koch-Weser, mencionado por Arora, Dhillo, Kumar y Sharma, 20I I).

\section{Metodología}

Se ensayaron diez dosis individuales del producto en estudio colocando las tabletas enteras en balones aforados de $100 \mathrm{~mL}$; a cada uno se le añadieron 10 gotas de agua destilada, permitiendo el contacto del agua con la tableta hasta lograr su desintegración. Posteriormente se le agregaron cerca de $60 \mathrm{~mL}$ de agua destilada a cada balón y se colocaron en baño ultrasónico por 10 minutos, hasta lograr la disolución total del principio activo. Luego se colocaron en baño de agua fría los balones y se aforaron con el mismo disolvente cuando estos se encontraban a temperatura ambiente; después de aforarlos, se filtró cada uno con papel de filtro cuantitativo número 2, para eliminar los excipientes insolubles.

Posteriormente se tomó una alícuota de $0,5 \mathrm{~mL}$ y se llevó a un balón de $100 \mathrm{~mL}$ con agua destilada, para obtener una concentración teórica esperada y aproximada de 7,5 mg/L. Se preparó una disolución madre de teofilina en agua destilada, con una concentración aproximada a $800 \mathrm{mg} / \mathrm{L}$ en un balón de $25 \mathrm{~mL}$, a partir de un patrón de pureza conocida, y se diluyó I mL en 100 mL con agua destilada, para obtener una concentración de $8 \mathrm{mg} / \mathrm{L}$. Por último, se midió la absorbancia del patrón y las muestras de teofilina en un espectrofotómetro UV/Vis, Perkin Elmer Lambda 20, utilizando agua destilada como blanco reactivo y se calcularon los miligramos de teofilina presentes en cada dosis ensayada y el porcentaje de lo etiquetado en cada caso.

\section{Resultados}

El análisis de uniformidad de contenido realizado a las tabletas de teofilina fabricadas por dos diferentes casas farmacéuticas de Costa Rica muestra poca variación en sus resultados. El estudio de la cantidad en miligramos de principio activo (teofilina) en las tabletas producidas por LISAN (media de $156,8 \mid$ $\mathrm{mg} / \mathrm{tab}$, desviación estándar de 3,15 mg/tab, $\mathrm{n}=10$ tabs) se muestra en el cuadro I. Los datos obtenidos son estadísticamente aceptables, ya que el coeficiente de variación es de $2,00 \%$, con un rango de 10,08 mg/tab y una desviación estándar de 3,15 mg/tab, y cada dosis ensayada se encuentra dentro del intervalo de $85 \%$ a $115 \%$ de lo etiquetado, según lo establece como criterio de aceptación la Farmacopea de Estados Unidos.

En el caso del análisis de las tabletas de la CCSS, mostrado en el cuadro 2 , se determinó la cantidad de teofilina (media de 154,07 mg/tab, desviación estándar de 3,62 mg/tab, $n=10$ tabs). Los datos obtenidos presentan un coeficiente de variación de 2,35\%, lo cual indica buena confiabilidad, además presentan un rango de $10,04 \mathrm{mg} /$ tab y una desviación estándar de 3,62 mg/tab; e igualmente cada dosis ensayada se encuentra dentro del intervalo de $85 \%$ a $\mid$ I $5 \%$ de lo etiquetado, según lo establece como criterio de aceptación la Farmacopea de Estados Unidos.

Con el objetivo de comparar ambos promedios, se realizó una prueba de hipótesis para la diferencia de dos medias, en la cual la hipótesis nula es que ambos promedios son iguales y la hipótesis alternativa es que son diferentes. Al realizar la prueba con un 99,99\% de confianza, no se obtuvieron suficientes evidencias para asegurar que ambas medias son diferentes, por lo tanto no se rechaza la hipótesis nula.

Lo anterior demuestra que ambos promedios son muy cercanos entre sí, entonces ambas casas farmacéuticas producen tabletas con cantidades de teofilina muy similares, como se observa en la figura 2.

Además, en ambos casos la variabilidad entre las tabletas analizadas es muy baja, tal y como se observa en la figura 3, donde vemos que el contenido de teofilina en las tabletas de ambas casas oscila entre $100 \%$ y $108 \%$ con respecto a lo etiquetado por tableta, con desviaciones estándar relativas inferiores al 2,5\% en ambos casos. Por lo tanto, podemos decir que el proceso productivo de las tabletas de teofilina empleado por ambos fabricantes es controlado y muy homogéneo.

\section{Discusión}

La uniformidad de contenido indica que las tabletas de la CCSS poseen una mayor exactitud con respecto a las de LISAN en cuanto a la cantidad del principio activo teofilina, aunque ninguna tableta, ni de LISAN ni de la CCSS, se encuentra fuera de las especificaciones de la Farmacopea de Estados Unidos. 
Cuadro I. Resultados de la uniformidad de contenido en tabletas de teofilina producidas por LISAN.

\begin{tabular}{|c|c|c|c|}
\hline Muestra & Absorbancia $( \pm 0,00 \mathrm{I})$ & Cantidad de teofilina $( \pm 3,15 \mathrm{mg})$ & $\begin{array}{c}\text { Porcentaje de lo } \\
\text { etiquetado }(\%)\end{array}$ \\
\hline 1 & 0,426 & 153,32 & 102,21 \\
\hline 2 & 0,422 & 151,88 & 105,25 \\
\hline 3 & 0,440 & 158,36 & 104,13 \\
\hline 4 & 0,434 & 156,20 & 103,41 \\
\hline 5 & 0,431 & 155,12 & 107,97 \\
\hline 6 & 0,450 & 151,96 & 105,57 \\
\hline 7 & 0,440 & 159,80 & 102,93 \\
\hline 8 & 0,444 & 154,40 & 105,81 \\
\hline 9 & 0,429 & 158,72 & $104,54 \%$ \\
\hline
\end{tabular}

Cuadro 2. Resultados de la uniformidad de contenido en tabletas de teofilina producidas por la CCSS.

\begin{tabular}{|c|c|c|c|}
\hline Muestra & Absorbancia $( \pm 0,00 \mathrm{I})$ & Cantidad de teofilina $( \pm 3,62 \mathrm{mg})$ & $\begin{array}{c}\text { Porcentaje de lo } \\
\text { etiquetado }(\%) *\end{array}$ \\
\hline 1 & 0,431 & 160,39 & 106,93 \\
\hline 2 & 0,406 & 151,09 & 100,73 \\
\hline 3 & 0,421 & 156,67 & 100,23 \\
\hline 4 & 0,404 & 150,35 & 103,70 \\
\hline 5 & 0,418 & 155,56 & 102,96 \\
\hline 6 & 0,415 & 154,44 & 101,47 \\
\hline 7 & 0,409 & 152,21 & 100,73 \\
\hline 8 & 0,406 & 151,09 & 100,23 \\
\hline 9 & 0,404 & 150,35 & 158,53 \\
\hline 10 & 0,426 & & \\
\hline \multicolumn{2}{|c|}{$*$ Promedio de porcentaje con respecto a lo etiquetado= $102,71 \%$} & \\
\hline
\end{tabular}

El promedio de porcentaje de lo etiquetado de teofilina para las tabletas de LISAN fue de $104,54 \%$, o sea 6,81 mg en promedio por encima de $150 \mathrm{mg}$, que era el valor etiquetado. Por otra parte, el promedio de porcentaje con respecto a lo etiquetado obtenido en las tabletas de la CCSS fue de 102,71\%, lo cual indica 4,07 mg en promedio sobre el valor etiquetado. Esto revela la buena calidad del proceso de manufactura implementado por ambas casas farmacéuticas.

Además, los análisis para cuantificar el principio activo de la teofilina se realizaron bajo los mismas condiciones, tanto para las tabletas de la CCSS como para las de LISAN. Con esto se garantizó una buena reproducibilidad del análisis, lo que conlleva a una mayor confiabilidad en los datos obtenidos. 


\section{Tableta vrs \% de lo etiquetado de teofilina}

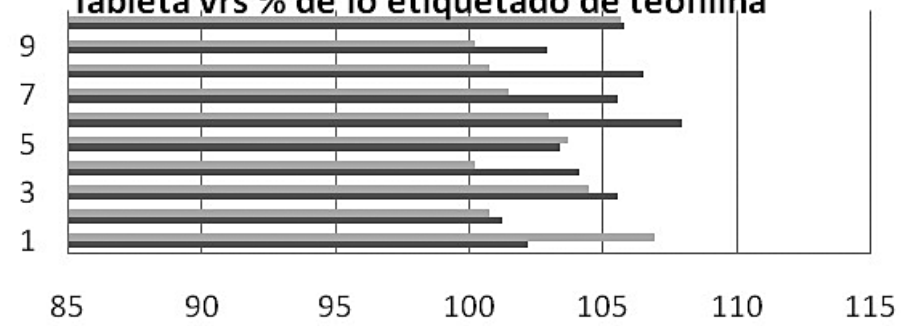

\begin{tabular}{|l|c|c|c|c|c|c|c|c|c|c|}
\cline { 2 - 10 } \multicolumn{1}{c|}{} & 1 & 2 & 3 & 4 & 5 & 6 & 7 & 8 & 9 & 10 \\
\hline$\square$ CCSS & 106, & 100, & 104, & 100, & 103, & 103 & 101, & 100, & 100, & 105, \\
\hline$\square$ LISAN & 102, & 101, & 105, & 104, & 103, & 108 & 105, & 106, & 102, & 105, \\
\hline
\end{tabular}

Figura 2. Comparación de uniformidad de contenido en tabletas de teofilina de dos casas farmacéuticas (LISAN y CCSS).

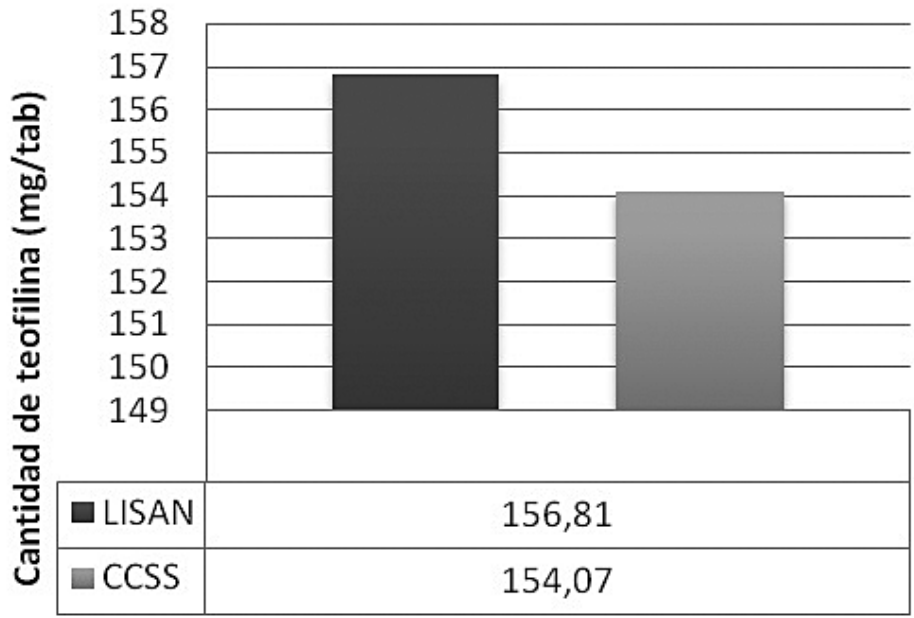

Figura 3.Variabilidad en el contenido de teofilina en tabletas de dos casas farmacéuticas (LISAN y CCSS).

Sin embargo, solo el promedio no es suficiente para garantizar que el principio activo se encuentre dentro de los límites aceptables, por lo cual se tomaron en cuenta otros parámetros, como la desviación estándar, el coeficiente de variación y el rango.

En cuanto a la desviación estándar como parámetro para medir la variación de los datos, los valores obtenidos fueron de 3, I 5 para LISAN y 3,62 para la CCSS; el coeficiente de variación (DSR) para ambos conjuntos de datos fue de 2,00\% para LISAN y
2,35\% para la CCSS, y la especificación establece que no debe ser mayor al $6 \%$.

Con respecto al rango de los datos, que nos indica qué tan alejado se encuentra el valor máximo del mínimo, el rango de los datos obtenidos en el análisis de las tabletas de LISAN fue de 10.08 mg, mientras que el de la CCSS fue de 10,04 mg. Considerando lo anterior, se puede afirmar que los datos presentan poca dispersión, lo que es lo más adecuado en un análisis de laboratorio y más 
si se trata de considerar la variabilidad propia del proceso productivo, como en este caso. Además, la especificación para la prueba va de $85 \%$ a II5\% de lo etiquetado para cada dosis ensayada, lo cual corresponde a un rango de $45 \mathrm{mg}$ en el caso de las tabletas analizadas que son de $150 \mathrm{mg}$ de principio activo, por lo cual lo obtenido fue muy bueno.

Una vez determinado el nivel de dispersión de los datos, se realizó la prueba de hipótesis, la cual evidenció que ambos promedios son estadísticamente iguales, ya que con un nivel de confianza de $99,99 \%$, no se encontró evidencia suficiente para afirmar que existe una diferencia significativa entre ambos promedios. Esto confirma que existe poca diferencia entre el valor obtenido para las tabletas de LISAN y para las de la CCSS.

\section{Consideraciones finales}

Los resultados individuales de las tabletas analizadas para los dos productos estudiados demuestran el cumplimiento de las especificaciones establecidas por la farmacopea para la prueba realizada, además de presentar resultados muy homogéneos.

La prueba de hipótesis para la diferencia de dos medias reflejó que no hay suficiente evidencia estadística para afirmar que las medias son diferentes, por lo que ambos promedios obtenidos son similares; además de que los desvíos relativos reflejan la baja variabilidad presentada en el contenido por tableta obtenida en los dos productos analizados.

La teofilina es una xantina ampliamente utilizada por vía oral e intravenosa en el tratamiento del asma y de los broncoespasmos. Además es una sustancia natural que existe en el té y está químicamente emparentada con la cafeína y la teobromina.

Además de sus efectos broncodilatadores, la teofilina también tiene propiedades inmunomoduladoras y antiinflamatorias y relaja el músculo liso bronquial así como los vasos pulmonares.
En los pacientes asmáticos, la teofilina reduce las respuestas de las vías aéreas a la histamina, metacolina, adenosina y alergenos. Sin embargo, la capacidad de la teofilina para controlar el asma crónica no se puede explicar a partir de su actividad broncodilatadora, que es relativamente débil. Las propiedades antiinflamatorias de la teofilina explican sus efectos en las reacciones tardías del asma.

Otros efectos extrapulmonares de la teofilina son los estimulantes sobre el sistema nervioso central, el aumento de la contractilidad del diafragma y la inhibición de las prostaglandinas. Además, el hecho de que la teofilina sea capaz de reducir la apnea del sueño en pacientes con insuficiencia cardíaca es una prueba de que debe existir algún mecanismo de origen central.

\section{Bibliografía}

Arora, S., Dhillon, V., Kumar,V. \& Sharma, A. (20I I). Use of simple spectrophotometric method for estimation of theophylline $(\mathrm{TH})$ in saliva amd urine of healthy human volunteer. International Journal of Pharma and Bio Sciences 2(3): 36-4I.

Bosch, C. \& Sanchez, F. (20/2). Recent applications in derivative ultraviolet/visible absorption spectrophotometry: 20092011. Microchemical Journal 106: I- 16.

Durán, D. (20 I I). Análisis fisicoquímico de productos farmacéuticos en las diferentes etapas del proceso de la industria farmacéutica. Universidad de Carabobo.

Johnson, I., Malathi, R., Prakash, H., Prathiba, J. \& Raghunathan, R. (20/2). Spectral Analysis of Naturally Occurring Methylxanthines (Theophylline, Theobromine and Caffeine) Binding with DNA. PLOS ONE 7(12): I- I |.

Kadam, V., Malke, S. \& Shidhaye, S. (2009). Validated stability indicating HPLC method for estimation of theophylline from a novel microsphere formulation. Asian Journal of Pharmaceutics 13-17.

Lavaut, M. \& Rodríguez, J. (2009). Validación del método de determinación de uniformidad de contenido en tabletas de dipirona de 300mg en el Laboratorio Farmacéutico Oriente. Revista Cubana de Química 21 (2): 66-69.

Ponce de León, L. \& Rojas, J. (2005). Desarrollo y validación de un método analítico (HPLC-RP) para la determinación de teofilina. Revista Colombiana de Ciencias QuímicoFarmacéuticas 28: 25-31. 